IJICIS, Vol.21, No.2, 140-154

DOI: 10.21608/ijicis.2021.73726.1087

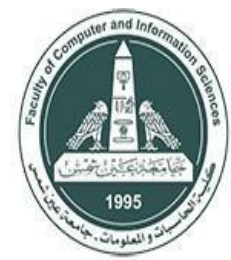

International Journal of Intelligent Computing and Information Sciences

https://ijicis.journals.ekb.eg/

\title{
INTELLIGENT SYSTEM FOR HUMAN AUTHENTICATION USING FUSION OF DORSAL HAND, PALM AND FINGER VEINS
}

\author{
Mona A. Ahmed \\ Computer Science Department, \\ Faculty of Computer and Information \\ Sciences, \\ Ain Shams University \\ Cairo, Egypt \\ eng.monaa_cs@hotmail.com
}

\author{
Mohamed .Roushdy \\ Computer Science Department, \\ Faculty of Computer and Information \\ Technology, \\ Future University, \\ New Cairo, Egypt \\ mohamed.roushdy@fue.edu.eg
}

\author{
Abdel-Badeeh M. Salem \\ Computer Science Department, \\ Faculty of Computer and Information \\ Sciences, \\ Ain Shams University \\ Cairo, Egypt \\ absalem@cis.asu.edu.eg
}

\begin{abstract}
Multimodal biometric systems roughly used to achieve extreme recognition accuracy. This paper reports a novel multimodal biometric system employing intelligent technique to authenticate human by fusion of dorsal hand, palm and finger veins pattern. We improved an image analysis technique to separate region of interest (ROI) from dorsal hand, palm and finger veins image. After separating ROI we construct a sequence of preprocessing steps to enhance dorsal hand, palm and finger veins images using Median filter, Wiener filter, Contrast Limited Adaptive Histogram Equalization (CLAHE) and Homomorphic filter to improve vein image. Our intelligent technique is based on the following intelligent algorithms, namely; principal component analysis (PCA) algorithm for feature extraction and $k$-Nearest Neighbors $(K-N N)$ classifier for matching operation. The database selected was Bosphorus Hand Vein Database, CASIA Multi-Spectral Palmprint Image Database V1.0 (CASIA database) and the Shandong University Machine Learning and Applications - Homologous Multi-modal Traits (SDUMLA-HMT). The accomplished result for the fusion of three biometric traits was Correct Recognition Rate (CRR) is $99.21 \%$ with False Reject Rate (FRR) 0.04\%.
\end{abstract}

Keywords: Biometric systems, pattern recognition, intelligent computing, image processing, machine learning.

\section{Introduction}

* Corresponding author: Mona A. Ahmed

Computer Science Department, Faculty of Computer and Information Sciences, Ain Shams University,Cairo, Egypt

E-mail address: eng.monaa_cs@hotmail.com 
A protected authentication system would confirm that only certified users are capable to approach or influence information. There are four means used in identification users; using what they know such as passwords and pins, using what they own which involve tokens and smart cards, using what they do which involves use of behavioral biometric and using what they possess which are the physiological biometrics [1].

Biometric authentication is a procedure of recognizing a person using physiological or behavioral traits. Physiological traits are Iris, DNA, hand, finger print and face behavioral traits are voice, signature, password, keystroke etc. Among all the authentication techniques present, biometrics is considered as the most reliable authenticators since they are unique to every individual and hard to get. The technology of Vein patterns as a type of biometric authentication was first intended in 1992. Vein pattern is the grid of blood carriers below an individual's skin layers. Vein patterns structure distinct and distinguishable patterns across various people and they remain the same irrespective of age. The patterns of blood veins are single to each person, even among twins. There are internal and external biometric systems. External include face, Iris, finger print based systems. Palm vein, finger vein, dorsal veins structure the internal biometric frameworks. Veins are intra-skin elements, consequently this feature makes the frameworks exceptionally secure, and they are not influenced by state of the external skin [2].Generally, biometric system operates in two modes explicitly:(i) verification mode in which biometrics can be used to prove an identity of person and (ii) identification mode in which biometrics can be used to decide an identity of person, even without that individual's information [3].

Hand vein technology operates by recognizing the vein prototypes (palm, dorsal hand and finger veins) in a hand of individual. When a hand of user is occupied over a scanner, a near infrared (NIR) beam plots the place of the veins. The red blood units existing in the veins absorb the beams and surface up on the map as black lines, while the remaining hand construction displays as white. This vein prototype is then checked against a preregistered prototype to authenticate the individual. As veins are internal in the body and have a variety of differentiating structures, attempts to forge an identity are awfully complicated, thus authorizing an extreme stage of safety [4].

Biometric authentication can be arranged into unimodal and multimodal biometric systems. Unimodal systems utilization single biometric character for recognition; and endures a few commonsense issues like non-all-inclusiveness, loud sensor information, intra-class variety, limited level of opportunity, inadmissible mistake rate, inability to-enlist and farce assaults. Along these lines, the exhibition of single biometric system should be improved. The multimodal biometric system used to solve the issues coming from unimodal biometric system. Multimodal biometric system utilizes distinctive biometric characteristics at the same time to verify an individual's personality. Multimodal biometric systems achieved robustness and high security of authentication [5].

This paper proposed a novel technique to authenticate user based on fusion of dorsal hand, palm and finger veins. Multimodal system used three separated database Bosphorus database, CASIA database and SDUMLA-HMT database. First, we extract region of interest (ROI) which contain important features. Then, many algorithms used to enhance the quality of images median filter, wiener filter, CLAHE and Homomorphic filter. Finally, Depended on PCA algorithm the system extract the important features which used by K-NN classifier to classify users.

The frame of the paper is structured as follows. Section 2 reports a broad analysis for the multimodal of recognition techniques and systems. We presented an indication of several fusion levels in multimodal biometrics systems in section 3. Section 4 explains briefly our methodology of the fusion of finger and hand vein system. Section 5 describes the explanation of the process of biometric system. The discussion of results is introduced in section6. Finally; section 7 presented conclusions and future work. 


\section{Analysis of Multi-modals Recognition Techniques and Systems}

There's many research used multi-model for authentication .In this section we introduced these studies divided to many fields such as modalities fused, method of feature extraction, methods of matching, level of fusion, database size and percentage of recognition to make analysis of it.

Table 1 Analysis of multimodal recognition techniques

\begin{tabular}{|c|c|c|c|c|c|c|c|}
\hline $\begin{array}{l}\text { Ref } \\
\text { No. }\end{array}$ & $\begin{array}{l}\text { Modalities } \\
\text { fused }\end{array}$ & $\begin{array}{c}\text { Feature } \\
\text { extraction } \\
\text { Method }\end{array}$ & $\begin{array}{l}\text { Matching } \\
\text { Method }\end{array}$ & $\begin{array}{l}\text { Fusion } \\
\text { levels }\end{array}$ & $\begin{array}{c}\text { Size of } \\
\text { Database }\end{array}$ & $\begin{array}{l}\text { Authentication } \\
\text { identification }\end{array}$ & $\begin{array}{l}\text { Percentage } \\
\text { of } \\
\text { recognition }\end{array}$ \\
\hline$[5]$ & $\begin{array}{l}\text { palm vein + } \\
\text { dorsal hand } \\
\text { vein }\end{array}$ & Proposed filter & $\begin{array}{l}\text { Euclidean } \\
\text { distance }\end{array}$ & $\begin{array}{c}\text { Feature } \\
\text { level }\end{array}$ & $\begin{array}{l}2400 \text { images } \\
250 \text { images }\end{array}$ & authentication & High FMR \\
\hline [4] & $\begin{array}{c}\text { finger vein } \\
+ \\
\text { fingerprint }\end{array}$ & Gabor filters & $\begin{array}{l}\text { Hamming } \\
\text { distance }\end{array}$ & score level & 6264 images & identification & $98.78 \%$ \\
\hline$[6]$ & Face +iris & $\begin{array}{c}\text { LBP } \\
\text { Daughman's }\end{array}$ & $\begin{array}{c}\text { LBPH Hamming } \\
\text { Distance }\end{array}$ & $\begin{array}{c}\text { decision } \\
\text { level }\end{array}$ & $\begin{array}{l}400 \text { images } \\
200 \text { images }\end{array}$ & authentication & $77 \%$ \\
\hline [7] & $\begin{array}{c}\text { Hand } \\
\text { geometry }+ \\
\text { palm veins }\end{array}$ & $\begin{array}{l}\text { proposed } \\
\text { algorithm } \\
\text { HOG } \\
\end{array}$ & SVM & $\begin{array}{c}\text { Feature } \\
\text { level }\end{array}$ & $\begin{array}{c}7.200 \\
\text { images }\end{array}$ & identification & $98.7 \%$ \\
\hline [8] & $\begin{array}{c}\text { Finger Vein } \\
+ \\
\text { Fingerprint }\end{array}$ & SMR & $\begin{array}{l}\text { Weighted sum } \\
\text { rule }\end{array}$ & score level & 1500 images & identification & $99.22 \%$ \\
\hline [9] & $\begin{array}{c}\text { Finger Vein } \\
+ \text { Hand } \\
\text { Vein }\end{array}$ & MLBP & IGMF & score level & $\begin{array}{l}3916 \text { images } \\
4846 \text { images }\end{array}$ & authentication & $98 \%$ \\
\hline [10] & $\begin{array}{l}\text { Palm print } \\
\text { and } \\
\text { Fingerprint }\end{array}$ & Gabor filters & $\begin{array}{l}\text { Euclidean } \\
\text { distance }\end{array}$ & $\begin{array}{c}\text { Feature } \\
\text { level }\end{array}$ & 250 images & authentication & $87 \%$ \\
\hline [11] & $\begin{array}{l}\text { Palm Print } \\
+ \text { Palm } \\
\text { Vein }\end{array}$ & Gabor filter & $\begin{array}{l}\text { Euclidean } \\
\text { distance }\end{array}$ & $\begin{array}{c}\text { Feature } \\
\text { level }\end{array}$ & 28 images & authentication & $100 \%$ \\
\hline [12] & $\begin{array}{c}\text { Hand } \\
\text { geometry + } \\
\text { Hand Vein }\end{array}$ & $\begin{array}{c}\text { K-curvature } \\
\text { VPE }\end{array}$ & $\begin{array}{l}\text { Euclidean } \\
\text { distance }\end{array}$ & score level & 1300 images & authentication & $99.94 \%$ \\
\hline [13] & $\begin{array}{c}\text { Palm Print } \\
+ \text { Palm } \\
\text { Vein }\end{array}$ & $\begin{array}{c}\text { Wavelet packet } \\
\text { tree }\end{array}$ & $\begin{array}{c}\text { K NN } \\
\text { Naive Bayes }\end{array}$ & $\begin{array}{c}\text { Feature } \\
\text { level }\end{array}$ & 2400 images & authentication & $95.95 \%$ \\
\hline [14] & $\begin{array}{c}\text { Finger Vein } \\
+ \text { Iris }\end{array}$ & $\begin{array}{l}\text { Current tracking } \\
\text { point }\end{array}$ & $\begin{array}{l}\text { Hamming } \\
\text { Distance }\end{array}$ & score level & $\begin{array}{l}120 \text { images } \\
140 \text { images }\end{array}$ & identification & $92.40 \%$ \\
\hline [15] & $\begin{array}{c}\text { face }+ \\
\text { finger veins }\end{array}$ & PCA and LDA & $\begin{array}{c}\text { Euclidean } \\
\text { distance }\end{array}$ & score level & $\begin{array}{l}210 \text { images } \\
105 \text { images }\end{array}$ & authentication & $91.4 \%$ \\
\hline [16] & $\begin{array}{c}\text { face }+ \\
\text { fingerprint }\end{array}$ & Gabor filter & PCA & $\begin{array}{c}\text { Feature } \\
\text { level }\end{array}$ & $\begin{array}{l}400 \text { images } \\
136 \text { images }\end{array}$ & authentication & $98.11 \%$ \\
\hline
\end{tabular}




\begin{tabular}{|c|c|c|c|c|c|c|c|}
\hline$[17]$ & $\begin{array}{c}\text { Iris }+ \\
\text { Finger Vein }\end{array}$ & SIFT & $\begin{array}{l}\text { SVM } \\
\text { SIFT }\end{array}$ & score level & $\begin{array}{l}756 \text { images } \\
756 \text { images }\end{array}$ & authentication & $98 \%$ \\
\hline
\end{tabular}

From the exploration of the above study, it can inform the following critical results;

1) Most models has very high rate to acceptability (accept to use) is hand (palm, dorsal hand and finger) and the low rate be in possession the iris model. Hand veins have extreme level of security and accuracy the reason of that it prevented internal human body so there is no influenced by state of the external skin. Hand veins are the best model to use because of high rate of acceptability, accuracy and security.

2) There are many algorithms used in researches based on the model used and the quality of images such as Gabor filter, LBP, median filter, PCA and SIFT algorithms. The most algorithms used in pattern veins are PCA, LBP and SIFT because these algorithms give most significant definite variation information in features vector.

3) There are many algorithms used in researches based on the model used and features extracted from images such as Euclidean distance, SVM, Hamming Distance, Naive Bayes and K-NN classifier. The most algorithms used in pattern veins are K-NN and Euclidean distance classifier. K-NN classifier work better because of it have a similar scale, few info factors, small calculation time and it has high accuracy.

4) There are many fusion levels feature, score and decision level. The best and most difficult degree to achieve is feature fusion; it obtained from several identifiers should be free and in the same estimate scale. Matching score level fusion is easy to obtain and combine matching scores of different biometrics but more complexity as these scores are from various modalities and depend on various scaling techniques. Fusion at decision level is very easy to implement and has high accuracy but it need more time than other level of fusion.

5) The biggest problem in the phase of multimodel biometric system is there are few databases available to research. BANCA, BIOSEC, BIOMET and XM2VTS provide voice, face, ace, fingerprint hand and signature databases. SDUMLAHMT database provide many image database such as iris, fingerprint, face and gait.

6) Percentage of recognition in multi-biometric systems that contain one or more trait vein has higher rate compared with other multi-biometric systems because of advantages of veins model accuracy and security.

\section{Intelligent Fusion Levels and Techniques in Biometric Systems}

H.S.Ali and M.I.Abdalla [18] have introduced an outline of multimodal biometrics and have advised several fusion levels. The levels of fusion recommended for multimodal systems are shown in Fig. 1 and explained below. 


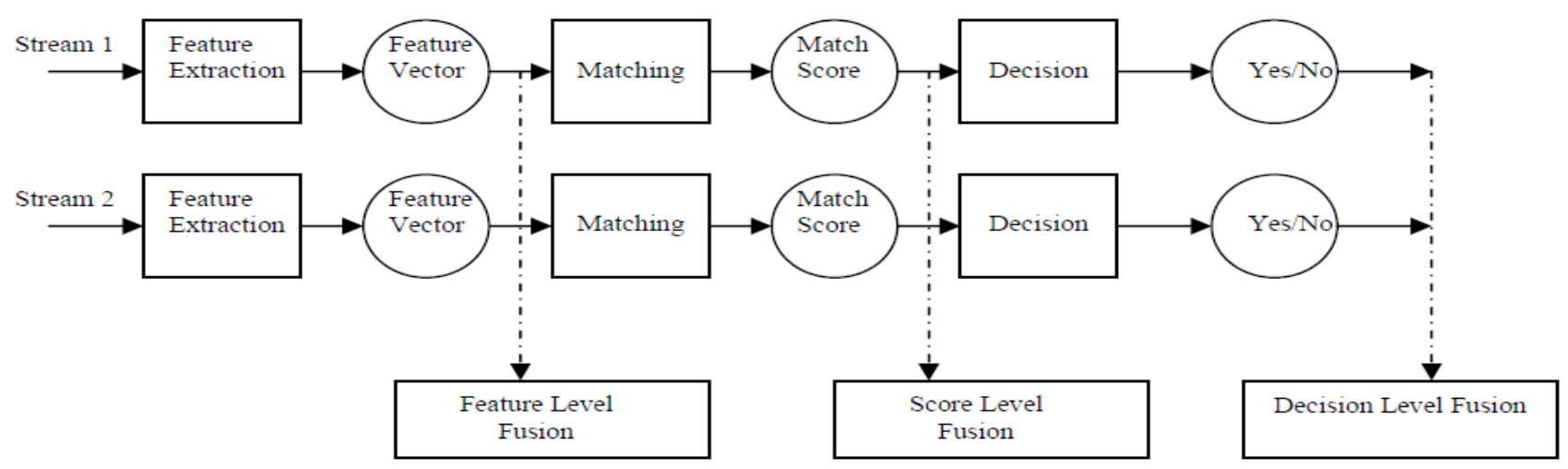

Figure 1 Fusion levels in multimodal biometric system

\section{A. Fusion at the Feature Extraction Level}

A feature vector compute using the data acquired from each sensor. As the features extracted from one biometric trait are independent of those extracted from the other, it is reasonable to concatenate the two vectors into a single new vector.

\section{B. Fusion at the Matching Score Level}

Feature vectors are generated separately for each sensor and are then compared to the enrollment templates which are stored independently for each biometric characteristic. Each system specifies a matching sore signifying the proximity of the feature vector with the template vector. These individual scores are lastly fused into a total score which is given to the decision module to establish the accuracy of the claimed identity.

\section{Fusion at the Decision Level}

An independent recognition decision is made for each biometric characteristic. These decisions are then merged into a final vote. The fusion procedure is achieved by a sequence algorithm such as OR, AND, etc. Also a common voting structure can be operated to compose the final decision.

\section{Proposed Methodology}

In our study, we present a proposed intelligent paradigm to authenticate personal based on fusion of dorsal hand, palm and finger veins. This paradigm is used to enhance the accuracy of vein authentication. Figure 2 present the methodology of the authentication model using fusion of dorsal hand, palm and finger veins biometrics. The proposed multi-modal biometric system involves of some various submodules, each of them presenting its private functionality. There are three sensor modules for dorsal hand, palm and finger veins gain. The captured data is managed to isolate a series of features. The extracted features are compared with the templates stored in enrollment stage, specifying a matching score. The decision making modules is a last module, which can organize either in identification or verification mode. 


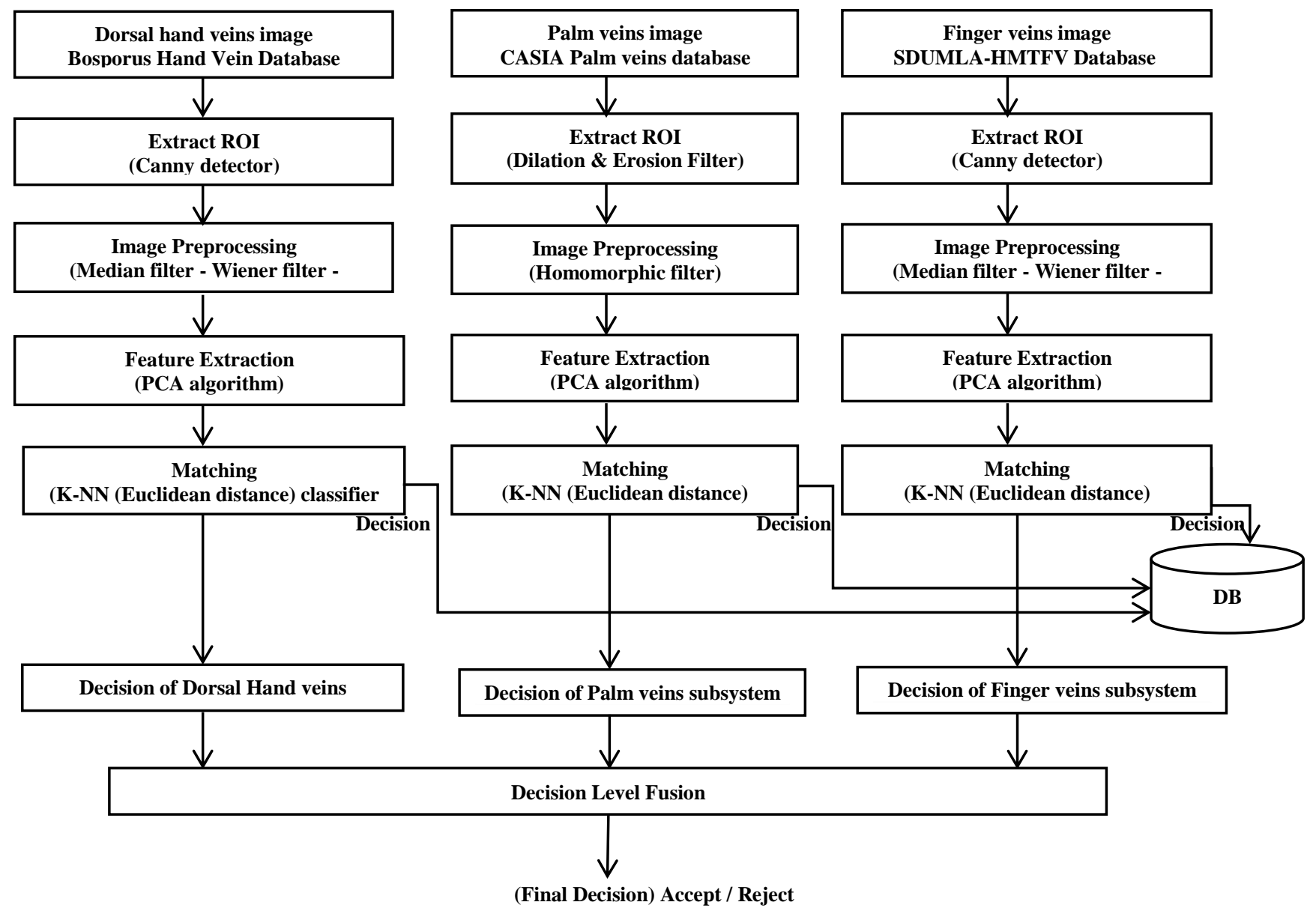

Figure 2 The methodology of the authentication model using fusion of dorsal hand, palm and finger veins biometrics

\section{Process of Biometric System}


In this partition, we describe explain the recognition process of finger and hand veins characteristics. The process of biometric system involves: image acquisition, extract ROI, preprocessing, feature extraction, matching, decision of each trait and fusion decision.

\subsection{Image Acquisition System}

\subsubsection{Dorsal Hand Veins Database}

The experiment informed in our study for dorsal hand vein authentication is the Bosphorus Hand Vein Database [19]. In our experiments, we used the images taken under normal conditions ( $\mathrm{N}$ : Normal). There are overall 600 images of 100 subjects distributed as: Three right-hand images and three left-hand images subject under normal conditions $(\mathrm{N})$.

\subsubsection{Palm Veins Database}

The experiment reported in our study for palm vein authentication is CASIA Multi-Spectral Palmprint Image Database V1.0 (CASIA database) [20]. CASIA database captured from 100 users in two data sessions (three images in each session) there is one month between the two sessions. Only the images captured under $850 \mathrm{~nm}$ and $950 \mathrm{~nm}$ wavelength illuminations used in our work because it focused on the vascular details.

\subsubsection{Finger Veins Database}

In our study, we used the SDUMLA-HMT Database [21].This database includes a multimodal data (face, finger vein, iris, finger print and gait) from 106 individuals. The first available open access database is SDUMLA-HMT. Each subject contains his/her code as index, middle and ring finger of both hands. The set of 6 fingers is repeated 6 times to obtain 6 finger vein images. Accordingly, finger vein database includes 3,816 images.

\subsection{Extract ROI}

\subsubsection{ROI of Dorsal Hand Veins}

The edge detection is an important process in many of the image processing algorithms. Significant property of the edge detection is the detection of the specific edges along with the great orientation of the object in the image [22]. In our technique the canny edge detector CED is used due to the following features, as show in figure 3.

1- Convert image to binary.

2- Boundaries from the binary image are detected by canny operator.

3- Valleys of hand between index and middle fingers and between little and ring fingers are detected. 
4- A geometrical technique is investigated to draw the line connecting the two keys points determined in the previous step and the line perpendicular to it.

5- A sub-image is detected and extracted as the ROI of hand vein image.

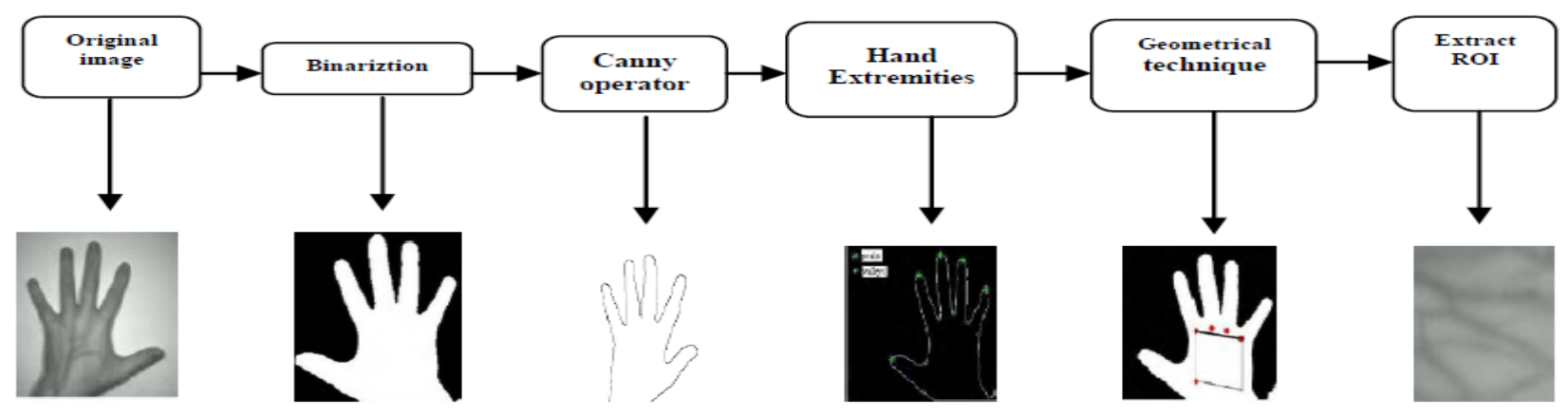

Figure 3The steps to detect ROI of dorsal hand veins

\subsubsection{ROI of Palm Veins}

To detect ROI we used Morphological operations to extract useful structural information from palm veins images. They are operated for edge detection, smoothing or extraction of other features. Dilation (expand objects by filling in small holes and connecting disjoint objects) and erosion (shrinks objects by eroding boundaries) are the two principal morphological operations [23, 24]. The proposed algorithm of ROI extraction of palm vein image includes 5 tasks, as show in figure 4.

1- Convert image to binary

2- Apply a 201*201 mask to estimate the area of the binary image that perfectly covers the whole region of palm.

3- Get one point that is the middle point of the palm by applying the dilatation filter again.

4- Operate the erosion filter on the constant square mask, this time to pick up square assigned at same point where $\mathrm{ROI}$ is placed in original image

5- Then observe xmin, ymin, length, and width of this square to crop ROI from actual image.

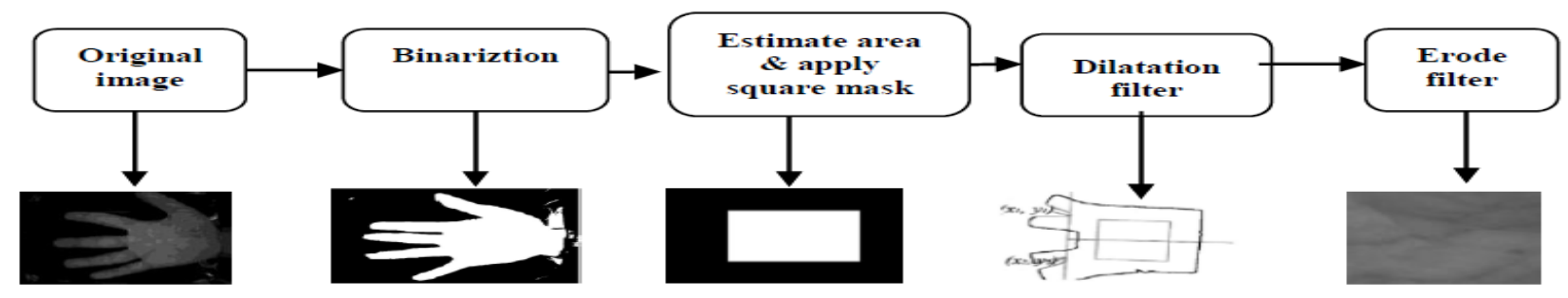

Fig. 4 The steps to detect ROI of palm veins

\subsubsection{ROI of Finger Veins}


To detect ROI, we used CED to extract useful structural information from finger veins images. The edge detection is an important process in many of the image processing algorithms. Significant property of the edge detection is the detection of the specific edges along with the great orientation of the object in the image [22]. The proposed algorithm of ROI extraction of finger vein image includes 3 tasks, as show in figure 5.

1- Convert image to binary.

2- Detect finger edge from the binary image using canny operator.

3- A sub-image is detected and extracted as the ROI of finger vein image.

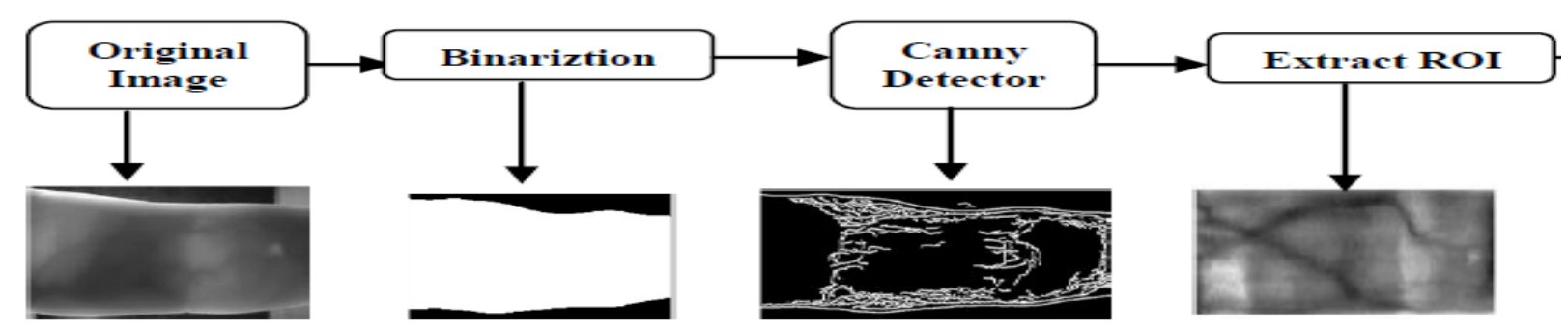

Figure 5 The steps to detect ROI of finger veins

\subsection{Image preprocessing}

\subsubsection{Preprocessing of Dorsal Hand Veins}

In this process, we need to enhance the hand veins images by reducing effect of noise. The proposed algorithm of preprocessing hand vein image includes the following steps done as show in figure 6.

1- Utilized median filter to original hand vein image for denoising.

2- 2D Wiener filter "Gaussian white noise" is applied to remove the influence of high level frequency noise.

3- Applied CLAHE filter to improve hand vein image.

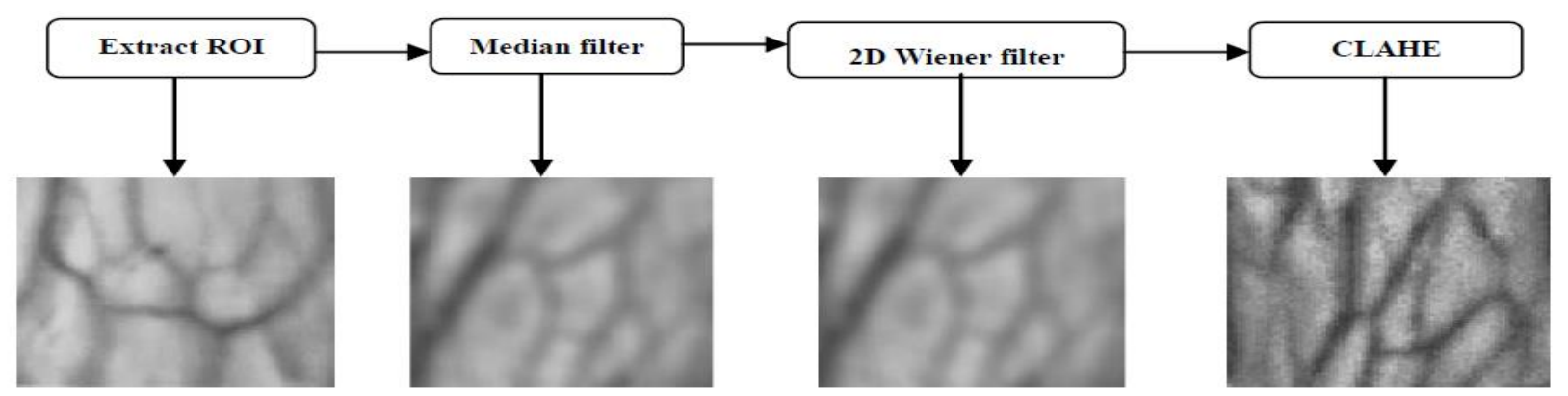

Figure 6 the steps of preprocessing of dorsal hand veins

\subsubsection{Preprocessing of Palm Veins}

Homomorphic filtering is a universal technique for image improvement and/or adjustment. It concurrently standardizes the brightness across an image and increases contrast. The function of 
homomorphic filter is expected to reduction the low frequency and arise the high frequency. Figure 7 illustrations the results of operated preprocessing step to enhance the image quality.

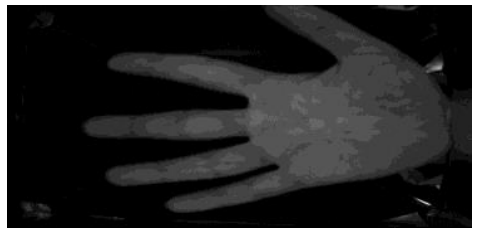

(a)

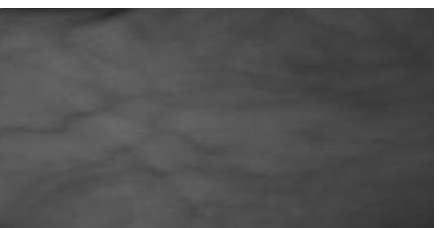

(b)

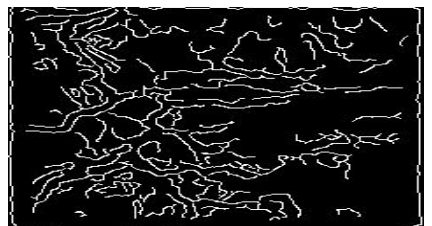

(c)

Figure 7 Illustration of image enhancement: (a) The original image (b) Extraction of ROI (c) Extraction of Palm vein pattern

\subsubsection{Preprocessing of Finger Veins}

In this process, we need to enhance the finger veins images by reducing effect of noise. The proposed algorithm of preprocessing finger vein image includes the following steps done as show in figure 8 .

1- Apply the median filter to reduce the black noise between vein pattern lines

2- Apply the wiener filter to remove effect of high frequency noises.

3- Apply the CLAHE filter to enhance contrast of vein image.

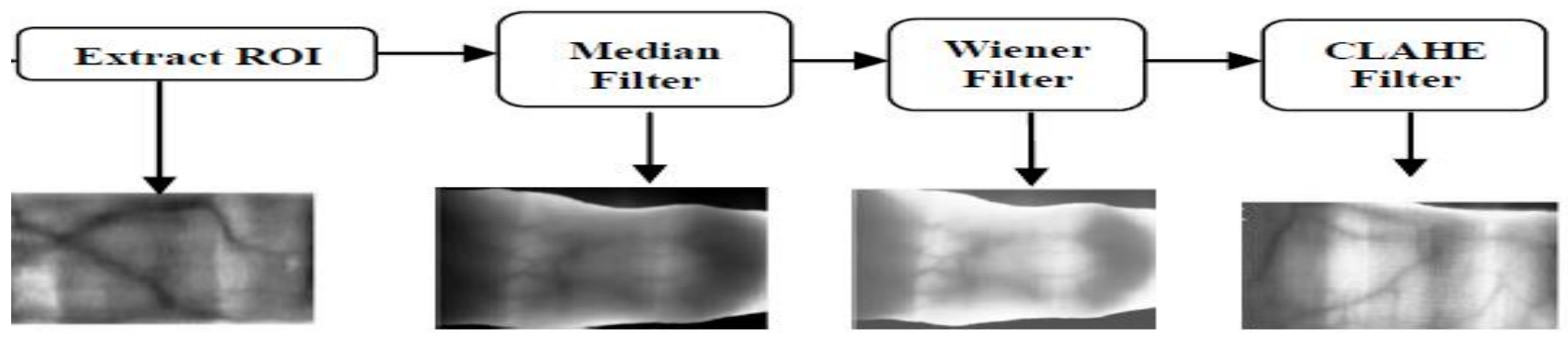

Figure 8 The steps of preprocessing of finger veins

\subsection{Feature Extraction}

In our system, we use principal component analysis (PCA) algorithm to extract features from images. A matching procedure is then used to locate the best match from the database to recognize the user. It converts several feasibly related factors into fewer new factors, called as principal components [25]. Since a digital image can be viewed as a two (or more) dimensional function of pixel values and represented as a 2D or 3D data array. More technical details can be found in [26]. Figure 9 shows Pseudo-code for PCA algorithm.

\footnotetext{
Input: $\mathrm{x}_{1}, \ldots, \mathrm{x}_{\mathrm{n}} \mathrm{d}$ length vector, $\mathrm{k}$

Output: Transform matrix R

BEGIN

$\mathrm{X} \Leftarrow \mathrm{n} \times \mathrm{d}$ data matrix with $\mathrm{xi}$ in each row;

$\bar{x} \Leftarrow \frac{1}{n} \sum_{i=1}^{n} x_{i} ;$

$\mathrm{X} \Leftarrow \operatorname{subtract} \overline{\boldsymbol{x}}$ from each row $\mathrm{x}_{\mathrm{i}}$ in $\mathrm{X}$;
} 
$\mathrm{COV} \Leftarrow \frac{1}{n-1} \mathrm{X}^{\mathrm{T}} \times \mathrm{X}$ Compute eigenvalue $\mathrm{e}_{1}, \ldots, \mathrm{e}_{\mathrm{d}}$ of $\mathrm{COV}$, and sort them;

Compute matrix $\mathrm{V}$ which satisfy $\mathrm{V}^{-1} \times \mathrm{COV} \times \mathrm{V}=\mathrm{D}$,

$\mathrm{D}$ is the diagonal matrix of eigenvalue of $\mathrm{COV}$;

$\mathrm{R} \Leftarrow$ the first $\mathrm{k}$ column of $\mathrm{V}$

END

Figure 9 Pseudo-code for PCA algorithm

\subsection{Matching}

In our system, we use the K-NN classifier. The nearest neighbor algorithm utilizes $\mathrm{K}$ nearest samples to the query image. Every one of these samples belongs to a known class Ci. The query image Iq is arranged to the class $\mathrm{CM}$ which has the most of events among the K samples [27]. The distance between two features can be compute depended on Euclidean distance. We used 5 different $\mathrm{k}$ values in our experiments to decide the best value of $\mathrm{k}-\mathrm{NN}$ : $\mathrm{k}=3, \mathrm{k}=5, \mathrm{k}=7, \mathrm{k}=9, \mathrm{k}=11$ and compared the accuracy of each $\mathrm{k}$ we find that a $\mathrm{K}=5$ get highest accuracy. Figure 10 illustrates the Pseudo-code for K-NN classifier.

Input: $\mathrm{D}=\{(\mathrm{x} 1, \mathrm{c} 1), \ldots,(\mathrm{xN}, \mathrm{cN})\}, \mathrm{x}=(\mathrm{x} 1, \ldots, \mathrm{xn})$ new instance to be classified

Output: $\mathrm{x}$ with the most frequent class

BEGIN

FOR each labeled instance (xi , ci )

Calculate d (xi , x)

Order d (xi, x) from lowest to highest, $(\mathrm{i}=1, \ldots, \mathrm{N})$

Select the K nearest instances to $\mathrm{x}$ : D_ $\mathrm{x}^{\wedge} \mathrm{k}$

Assign to $\mathrm{x}$ the most frequent class in $\mathrm{D} \_\mathrm{x}^{\wedge} \mathrm{k}$

END

Figure 10 Pseudo-code for K-NN classifier

\subsection{Fusion decision}

Our study multi-modal biometric system depend on three disparate modules: dorsal hand veins, Palm veins and finger veins for authentication. The fusion technique embraced at the decision level is post classification method and it follows the AND rule. This sequential coordinating with approach gives the chance of getting every one of the characteristics to decide whether user is certified or an impostor. The user is considered certified if module output is YES with score $[1,1,1]$ and when score otherwise this interval module output is No and the user considered an impostor. We used AND rule because of that 
comparative analysis shows that AND rule based decision fusion achieve higher performance as compare to OR rule based methods [28].

\section{Results and discussion}

In this portion we describe the result of each system independently and the result of fusion of three traits dorsal hand, palm and finger vein recognition. Because of the different organization of the databases, it became impossible to use any automatic method to merge them together, so we merge them manually. The experiment includes two phases training and recognition phases. In training phase, features of the training samples are estimated and saved in a database template. In the authentication phase, the input vein features are fixed and then matched by using K-NN matching classifier. After that, these features are matched with the stored template to get the recognition result. We do our experiment by split the database to 5 Cases as table 2 shows.

Table 2 Data base for 5 cases

\begin{tabular}{|c|c|c|}
\hline Case No. & Training & Testing \\
\hline 1 & One image for every person(100 images) & Five images for every person (500 images) \\
\hline 2 & Two images for every person $(200$ images $)$ & Four images for every person(400 images) \\
\hline 3 & Three images for every person $(300$ images $)$ & Three images for every person(300 images) \\
\hline 4 & Four images for every person $(400$ images $)$ & Two images for every person $(200$ images $)$ \\
\hline 5 & Five images for every person $(500$ images $)$ & One image for every person $(100$ images $)$ \\
\hline
\end{tabular}

By applying the PCA algorithm with K-NN (Euclidean distance) the results are 98.5\%, 100\%, 100\% and $100 \%$ for all training cases in dorsal hand, palm, finger veins and fusion of dorsal hand, palm and finger veins. Testing result of every case indicated in table 3 and figure 9 . We have two potential results, the first result is where the user that is unauthorized which means that his/her template is not found in the database, and the other result is the user is authorized, i.e. a template similar to his/her is found in the database. The experimental results displayed that the results of recognition Correct Recognition Rate (CRR) are $91.2 \%, 95.20 \%, 94.6 \%$ and for the fusion of 3 trait is $99.21 \%$ with False Reject Rate (FRR) $0.04 \%$. Depend on this experimentation, it was advised that recognition based on authentication by fusing the dorsal hand, palm and finger veins, accomplishes better than typical recognition technique. Hence this method can be successfully used for recognition.

Table 3 The testing results for each case

\begin{tabular}{|c|c|c|c|c|}
\hline \multirow{2}{*}{ Case No. } & & \multicolumn{3}{|c|}{ CRR } \\
\cline { 2 - 5 } & Dorsal hand & Palm & Finger & $\begin{array}{c}\text { Fusion Dorsal Hand + Palm } \\
\text { +Finger }\end{array}$ \\
\hline 1 & $\mathbf{8 9}$ & 94 & 93 & 98.7 \\
\hline 2 & 90 & 94 & 94 & 98.9 \\
\hline 3 & 92 & 95 & 95 & 98.9 \\
\hline 4 & 92 & 96 & 95 & 99.7 \\
\hline 5 & 93 & 97 & 96 & 99.85 \\
\hline
\end{tabular}




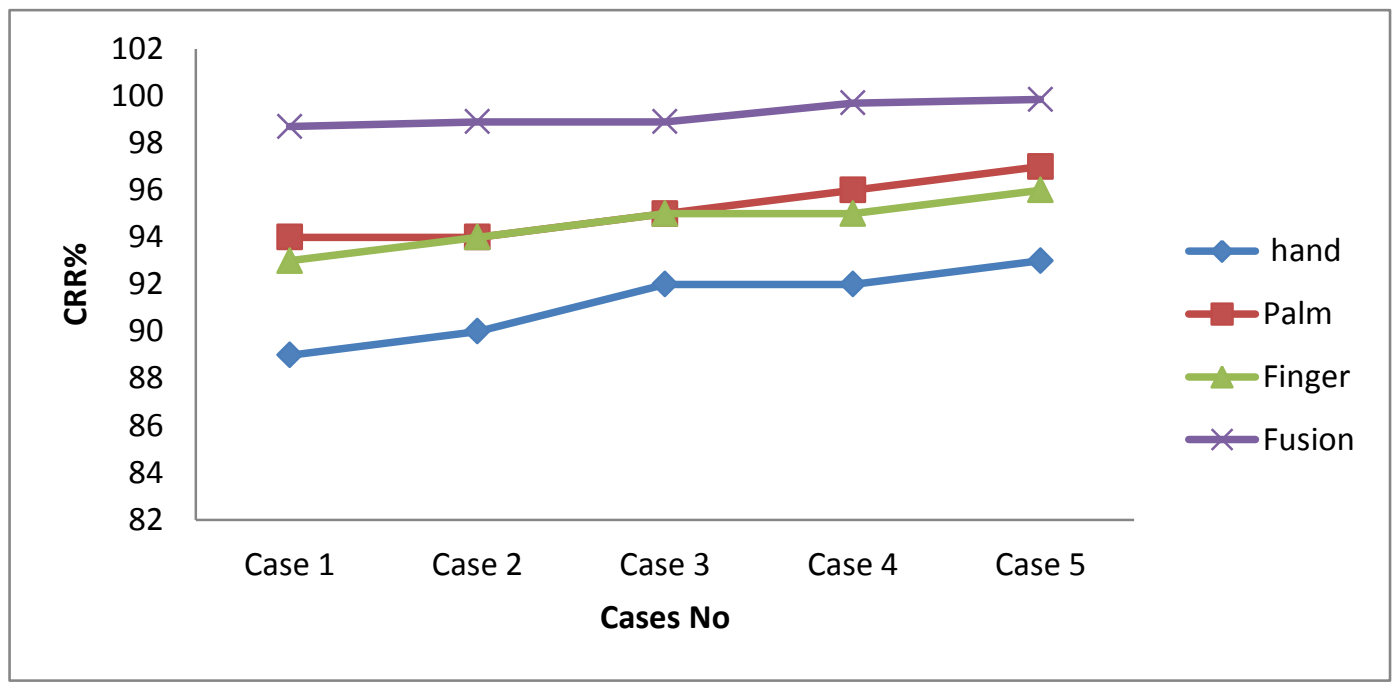

Figure 9 Result of cases

\section{Conclusion and Future Work}

In this paper, we have advanced a new practical and intelligent system for biometric recognition depends on fusion of dorsal hand, palm and finger veins. The system involves of the following steps: Image capture, determining the region of interest and pre-processing, extracting the vein pattern features and recognition. We proposed an original method depend on the principal component analysis (PCA) algorithm to extract features and using K-NN (Euclidean distance) matching classifier in matching. In this paper, a complete biometric system constructed fusion of finger and dorsal hand veins has been developed. We intended an original method based on the PCA algorithm to extract features and using KNN classifier in matching. The experimental results present that the result of recognition CRR is $99.21 \%$.Hence this method can be successfully used for recognition. The vein pattern recognition can progressed in an ideal way using the method recommended in this study which is precise, uncomplicated, concrete and rapid.

In our opinion, this developed improvement increases the usefulness and usability of this efficient technique, especially as regards its application in all security tasks and domains. Future work may involve utilizing this algorithm on fusion of other multimodel biometric system.

\section{References}

1- Mubeen Fatima, "Securing the Biometric template: A Survey", International Journal of Computer Applications, Vol.160, NO. 2, pp.1-8, February 2017.

2- Sumit Kulkarni , Manali Pandit, "Biometric Recognition System based on Dorsal Hand Veins", International Journal of Innovative Research in Science, Engineering and Technology(IJIRSET), Vol. 5, Issue 9,pp. 18899-18905, September 2016.

3- Laxmi Menaria, Kalpana Jain, “A Survey on Biometric Template Protection”, International Journal of Scientific Research in Computer Science, Engineering and Information Technology (IJSRCSEIT ),Vol. 2 ,Issue 2,pp.995-999, 2017. 
4- Shruthi.B.M, Pooja Mohnani, Mallinath, Ashwin.R.G, "Multimodal Biometric Authentication Combining Finger Vein and Finger Print", International Journal of Engineering Research and Development, Vol.7, No.10,PP.43-54, July 2013.

5- S.Bharathi and R.Sudhakar, "Biometric recognition using dorsal and palm vein images", International Journal of Advanced Engineering Technology, Vol. 7, No.2, pp.415-419, June, 2016.

6- E. Stefani and C. Ferrari, "Design and Implementation of a Multi-Modal Biometric System for Company Access Control", 2nd International Conference on Data Compression, Communication, Processing and Security (CCPS), pp.1-10, 2016.

7- L. E.Christo and A. Zimmer, "Multimodal biometric system for identity verification based on hand geometry and hand palm's veins", Communication papers of the Federated Conference on Computer Science and Information Systems, Vol. 13,pp. 207-212,2017.

8- R. Raghavendra ,Kiran B Raja, J. Surbiryala and C. Busch, "A low-cost multimodal Biometric Sensor to capture Finger Vein and Fingerprint", IEEE International Joint Conference on Biometrics, December 2014.

9- R. B. Trabelsi, A. D. Masmoudi and D. S. Masmoudi, “A New Multimodal Biometric System Based on Finger Vein and Hand Vein Recognition", International Journal of Engineering and Technology (IJET), Vol 5, No 4 ,pp.3175-3183,Sep 2013.

10- M. D Dhameliya and J. P Chaudhari, "A Multimodal Biometric Recognition System based on Fusion of Palmprint and Fingerprint", International Journal of Engineering Trends and Technology (IJETT) - Vol.4, No.5, pp.1908-1911, May 2013.

11- J. A. Siddharth, A.P. H. Prabha, T.J. Srinivasan and N. Lalithamani, "Palm Print and Palm Vein Biometric Authentication System", Artificial Intelligence and Evolutionary Computations in Engineering Systems, Advances in Intelligent Systems and Computing book,2017.

12- GiTae Park and Soowon Kim, "Hand Biometric Recognition Based on Fused Hand Geometry and Vascular Patterns", US National Library of Medicine National Institutes of Health, pp.2895-2910, February 2013.

13- V.Usharani,S.V.Saravanan, "Multi modal biometrics using palmprint and palmvein", Journal of Theoretical and Applied Information Technology, Vol. 67 ,No.1,pp.177-185, Sep.2014.

14- F. E. Mohammed, E. M. ALdaidamony and A. M. Raid, "Multi Model Biometric Identification System: Finger Vein and Iris", Vol.4, No4, pp.50-55, Sep. 2014.

15- M.I.Razzak, R.Yusof and M. Khalid, "Multimodal face and finger veins biometric authentication", Scientific Research and Essays, Vol. 5, No.17, pp. 2529-2534, Sep.2010.

16- Ayodeji S. Makinde, Yaw Nkansah-Gyekye and Loserian S. Laizer, "Enhancing the Accuracy of Biometric Feature Extraction Fusion Using Gabor Filter and Mahalanobis Distance Algorithm", International Journal of Computer Science and Information Security (IJCSIS), Vol.12, No.7, pp. 4148, July 2014.

17- F.E.Mohammed ,E.M.ALdaidamony and A.M.Raid, "Iris And Finger Vein Multi Model Recognition System Based On Sift Features", International Journal of Intelligent Computing and Information Science(IJICIS), Vol.15, No.1,pp.,15-24, JAN.2015.

18- H.S.Ali and M.I.Abdalla, "Score-Level Fusion for Efficient Multimodal Person Identification using Face and Speech", International Journal of Computer Science and Information Security (IJCSIS), Vol.9, No.4, pp.48-53, April 2011.

19- http://bosphorus.ee.boun.edu.tr/hand/Home.aspx

20- http://www. cbsr.ia.ac.cn/MS_Palmprint.

21- http://www.mla.sdu.edu.cn/sdumla-hmt.html . 
22- Jamil A. M. Saif, "Tuning of Canny Image Edge Detection", International Journal of Computer Science and Information Security (IJCSIS), Vol. 15, No. 11, ,pp.184-187, November 2017.

23- Ravi .S and A M Khan, "Morphological Operations for Image Processing: Understanding and its Applications", National conference on VLSI, signal processing \& communications NCVSCOMS2019, pp. 17-19, 2019.

24- M. Van Droogenbroeck and M. Buckley, "Morphological erosions and openings: Fast algorithms based on anchors", Journal of Mathematical Imaging and Vision, pp1-35, 2005.

25- Swathi $\mathrm{P}$ and Karunakar Pothuganti, "Overview on principal component analysis algorithm in Machine learning", International Research Journal of Modernization in Engineering Technology and Science(IRJMETS),Vol. 2, No. 10,pp. 241-246, 2020 .

26- AlešHladnik, "Image Compression and Face Recognition: Two Image Processing Applications of Principal Component Analysis", International Circular of Graphic Education and Research, No. 6, 2013.

27- Muhammad NaufalMansor,SazaliYaacob, HariharanMuthusamy, ShafrizaNishaBasah,Shahrul Hi-fi Syam bin Ahmad Jamil,MohdLutfiMohdKhidir,MuhammadNazriRejab,KuMohdYusri Ku Ibrahim,Addzrull Hi-fi Syam bin Ahmad Jamil,Jamaluddin Ahmad ,Ahmad KadriJunoh,“ PCABased Feature Extraction and k-NN algorithm for Early Jaundice Detection", International Journal of Soft Computing And Software Engineering (JSCSE),Vol.1,No.1,pp25-29,2011.

28- Suneet Narula Garg, Renu Vig and Savita Gupta, "Analysis of Decision Level Fusion in Multimodal Biometrics using Iris and Fingerprint", 3rd International Conference on Electrical, Electronics, Engineering Trends, Communication, Optimization and Sciences (EEECOS),pp. 406416, August 2017. 